CORRESPONDENCE

\section{Fluoxetine oral administration increases intraocular pressure}

EDrToR,-Fluoxetine is a widely prescribed antidepressant which acts as a selective inhibitor of neuronal serotonin uptake. ${ }^{1}$ Recently, $\mathrm{Ahmad}^{2}$ reported on a case of acute narrow angle glaucoma due to fluoxetine administration. For this purpose, we verified the intraocular pressure (IOP) variations in 20 consecutive depressed outpatients (five males and 15 females, age range 33-47 years) to whom fluoxetine therapy was first prescribed. All patients received a complete ophthalmic examination to exclude the pre-existence of both acute and chronic glaucoma. Patients with systemic diseases other than affective disorders were excluded from the study.

After at least 12 hours of fasting, in early morning $(8 \mathrm{am})$ subjects were assigned to either $20 \mathrm{mg}$ of oral fluoxetine or placebo in a randomised crossover double blind fashion. The alternative treatment was given 1 week later, a time lag considered sufficient for a single dose fluoxetine washout. ${ }^{3}$ Intraocular pressure was recorded (with a Goldmann tonometer mounted on a Haag-Streit slitlamp) at baseline and hourly up to 12 hours, both when patients were given fluoxetine and when they were given placebo.

All patients showed a significant increase in IOP 2 hours after oral administration of fluoxetine and 8 hours later some patients still exhibited higher IOP values (Fig 1); however, when patients received placebo there were no significant changes in IOP.

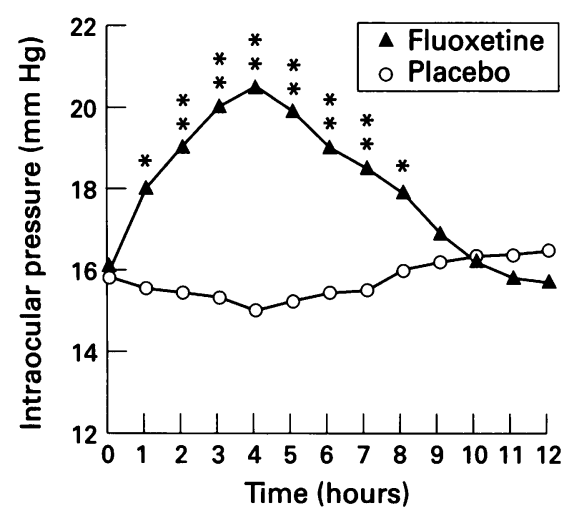

Figure 1 Intraocular pressure variations after fluoxetine and placebo oral administration. All values are mean plus or minus $S D$. Paired

Student's $t$ test was used for statistical analysis. ${ }_{\star_{p}}{ }^{*}<0.05 ;{ }^{*} p<0.01$ (treated versus untreated).

The mechanism by which fluoxetine in creases IOP might be due to the inhibitory effect on serotonin uptake. In fact serotonin, when injected into the anterior chamber, produces a significant increase in $\mathrm{IOP}^{4}$ and ketanserin, an agent with serotonergic blocking properties, reduces IOP both in animals and in humans. ${ }^{5}$ These data suggest that ophthalmic examination might be included in the protocol of depressed patients given fluoxetine therapy because this drug might raise intraocular pressure.
LEONARDO MASTROPASQUA Eye Clinic, Gabriele D'Annunzio University of Chieti

LUCA STEARDO NUNZIO TESTA Department of Neurology, Federico II University of Naples, Via S Pansini, 580131 Napoli, Italy

1 Wong T, Bymaster FP, Horng JS, Molloy BB. A new selective inhibitor of uptake of serotonin into synaptosomes of rat brain: 3-(ptrifluoromethy-phenoxy)-N-methyl 3 -phenyl-
propylamine. $f$ Pharmacol Exp Ther 1992;193:804-11.

2 Ahmad S. Fluoxetine and glaucoma. Ann Pharmacother 1991;25:436.

3 Malcolm L. Fluoxetine efficacy vs comparative drugs: an overview. Br f Psychol 1988;153:51-8.

4 Osborne NN, Tobin AB. Serotonin accumulating cells in the iris-ciliary body and cornea of various species. Exp Eye Res 1987;44:731-46.

5 Costagliola C, Iuliano G, Rinaldi M, Russo V, Scibelli G, Mastropasqua L. Effect of topical ketanserin administration on intraocular pressure. Br f Ophthalmol 1993;77:344-8.

\section{Visual screening programme for preschool children}

EDIToR,-I welcome Williamson et al's recent article $^{1}$ and your editorial ${ }^{2}$ suggesting that school vision screening may be the optimum method of detecting amblyopia in children in some circumstances. I would support the view that a larger number of children could be screened. In Glasgow, in the north west area, covering approximately the same geographical area as that quoted in Williamson et als study of preschool orthoptic screening, $95 \%$ of children enrolled in mainstream school in primary one were screened during the session 1994-5.

However, in the same year there was a significant difference between the number of new visual defects detected (unilateral visual acuity $6 / 9$ or poorer using a single letter test) in schools in the sector of the city where preschool orthoptic screening was being carried out-131/3235 (4\%)-compared with that where there was no preschool screening (398/3644 (11\%)).

The quality of school vision screening in Glasgow is being studied at present and improvement is actively being sought. Only once satisfactory standards can be demonstrated, in addition to the ability to screen a large proportion of the population, can it be argued that this should stand alone as the sole method of detecting amblyopia while it is still amenable to treatment.

KATHERINE M SPOWART Yorkhill NHS Trust

1 Williamson TH, Andrews R, Dutton GN, Murray G, Graham N. Assessment of an inner city visual $\mathrm{G}$, Graham N. Assessment of an inner city visual screening programme for presch

2 Elston J. Preschool visual screening. [Editorial] $\mathrm{Br}$ f Ophthalmol 1995;79:1063-4.

\section{Reply}

EDITOR,-The communication from our colleague, Dr Spowart, raises a statistic of interest-that is, an apparent but unsubstantiated reduction in the proportion of children with poor vision in areas of the north west of Glasgow with preschool screening. The $11 \%$ prevalence of children at school with $6 / 9$ vision or less (using singles testing) in the unscreened population is similar to the proportion of preschool children in our survey with this level of visual acuity at screening $(9.2 \%$, also with singles testing). Although the screening programme may have reduced the amblyopia somewhat we feel unable to take the credit for reducing the rate to $4 \%$. Refrac tion of the patients at the first visit improved the visual acuity in a proportion (78.3\% remained with $6 / 9$ or worse, Snellen acuity, after spectacles) but the proportion remained similar after the first year of hospital treatment - that is, when they were at school $(77.8 \%)$. We therefore cannot explain such a difference in the screened and unscreened populations of Dr Spowart especially when only $57 \%$ of the total population of children attended our screening services. Was the demographic constitution of her two populations different? Did the advertising of visua disability via preschool screening cause parents to seek advice from others-for example, optometrists? The questions remain. We advocate school screening by personnel specifically trained because of the increased attendance. Perhaps school nurses could be employed thereby allowing redeployment of orthoptists to refract and treat the increased numbers of children detected.

TOM H WILLIAMSON GORDON N DUTTON GORDON MURRAY ROSEMARY ANDREWS Tennent Institute of Ophthalmology, Glasgow

\section{BOOK REVIEW}

Colour Atlas of Scleritis. By P Watson, J M Ortiz. Pp 122. £55. London: Mosby Wolfe, 1995.

The authors have complied an exceptional illustrated documentation of scleral inflammation. The book is a great pleasure to read and, with the authors' selection and great variety of cases, gives the reader the full spectrum of scleral inflammation. The authors have provided a thorough description of the diagnosis and management of scleritis which includes understanding the normal anatomy and vasculature of both the conjunctival and episcleral vessels. Throughout the book they describe examples of scleritis and associated scleral inflammation using fluorescein angiography which beautifully illustrates the vascular changes that occur in the various forms of scleritis where examples of the diagnostic power of ultrasonography in these conditions are shown.

It is certainly a very useful and educational book to have in any department. If there is a criticism it is perhaps in the discussion of the management of scleritis, which would have benefited from an appraisal of the underlying immunopathology and greater details of why immunosuppressive regimes are used. Although I am sure it was not in their remit, documentation of case series with this form of treatment or reference to it would be of use to readers so that they can gain understanding of the success of such treatment.

Overall this is one of the clearest examples of presenting scleritis in an illustrated form available today. 


\section{LETTERS}

If you have a burning desire to respond to a paper published in the BJO, why not make use of our "rapid response" option? Log on to our website (www.bjophthalmol.com), find the paper that interests you, and send your response via email by clicking on the "eletters" option in the box at the top right hand corner.

Providing it isn't libellous or obscene, it will be posted within seven days. You can retrieve it by clicking on "read eletters" on our homepage. whether to also publish it in a future paper issue.

\section{Surgically induced diffuse scleritis: comparison of incidence in phacoemulsification and conventional extracapsular cataract extraction}

Surgically induced diffuse scleritis (SIDS) is recognised but less well reported cause of pain and reduced vision following cataract surgery.'

We have previously reported on complications of conventional extracapsular cataract extraction in which SIDS was the second most common. ${ }^{1}$ Recently, we conducted an audit of patients who underwent phacoemulsification cataract extraction to compare the incidence of SIDS in these patients relative to that found in the ECCE group.

\section{Methods and results}

From a computerised departmental database, 666 consecutive patients who had undergone phacoemulsification cataract surgery with intraocular lens (IOL) implantation under a single consultant firm were identified. The case notes were examined and all postoperative complications arising within the first 3 months were documented. The patients preoperative ophthalmic and general medical histories were also recorded to identify additional risk factors. The results of the study were compared with those from a previously
The editors will decide as before

published retrospective study of 682 consecutive patients who underwent conventional extracapsular cataract surgery (ECCE) under the same consultant firm at a time where ECCE was the preferred technique. ${ }^{1}$

Final visual acuities reached $6 / 12$ or greater in $80 \%$ of phacoemulsification patients and $67 \%$ of ECCE patients. The commonest complications occurring in both groups and in the National Cataract Surgery Survey (NCSS) 1997-8 are listed in Table 1.

\section{Comment}

Ten $(1.5 \%)$ of the patients who underwent phacoemulsification cataract extraction (including one whose procedure was combined with trabeculectomy) were diagnosed with SIDS. This was approximately half the proportion, $(2 \mathrm{l}=3.1 \%)$ of the ECCE group, but was the second commonest complication in both groups of patients. However, it does not feature in the list of postoperative complications reported in the National Cataract Surgery Survey 1997-8. ${ }^{2}$ SIDS has previously been described as an underdiagnosed clinical entity ${ }^{1}$ and it may be failure to recognise it which explains its absence from the national statistics.

Patients who had undergone previous ipsilateral iris surgery (trabeculectomy, Scheie's procedure, or YAG iridotomy) appeared to be at increased risk, although this only attained statistical significance in the phacoemulsifcation group $(\mathrm{p}=0.01)$. General anaesthesia was associated with higher statistical risk for ECCE patients only $(\mathrm{p}=0.009) .{ }^{1}$ It is unclear why this should have been the case. Intraoperative complications did not increase the risk of developing SIDS, and there was no association with concurrent ocular or systemic disease. The mean age of the SIDS patients in the ECCE group was approximately 11 years younger than that in the phako group (62.5 $\mathrm{v}$ 73.6).

It is our practice to take a thorough history from the postoperative patient. Severe pain, especially that waking the patient from sleep, is a common feature. Examination of the patient both on the slit lamp and in daylight is conducted, the latter to recognise the characteristic violaceous injection of the scleral vasculature. B-scan ultrasonography is performed to measure scleral thickness. Relative thickening compared to the contralateral eye or absolute thickness greater than $1.8 \mathrm{~mm}$ supports the diagnosis. Affected patients usually show a favourable response to a combination therapy with oral non-steroidal anti-

Table 1 Postoperative complications requiring intervention

\begin{tabular}{llll}
\hline Complication & Phako (\%) & ECCE (\%) & NCSS (\%) \\
\hline Astigmatism * & - & 9.5 & \\
Cystoid macular oedema & 1.95 & - & 0.05 \\
SIDS & 1.5 & 3.1 & - \\
Uveitis & 1.35 & 2.3 & 5.6 \\
Posterior capsule opacification & 0.75 & 1.9 & 0.4 \\
Endophthalmitis & 0.6 & $<1$ & 0.03 \\
Corneal decompensation & 0.3 & - & - \\
Subluxed IOL & 0.15 & $<1$ & 0.1 \\
Iris prolapse/wound dehiscence & 0.15 & $<1$ & 1.2 \\
Hyphaema & 0.15 & - & 1.1 \\
\hline
\end{tabular}

*Astigmatism requiring suture removal. inflammatory agents, ${ }^{3}$ topical steroids, and a topical cycloplegic. SIDS should be considered in the differential diagnosis of a painful red eye postoperatively. Prompt diagnosis and appropriate therapy lead to early resolution of SIDS and improved visual outcome.

J Sen

St Paul's Eye Unit, Royal Liverpool University Hospital, Prescot Street, Liverpool L7 8XP, UK

G G Kamath, L G Clearkin

Department of Ophthalmology, Arrowe Park Hospital, Wirral CH49 5PE, UK

Correspondence to: Dr Sen

Accepted for publication 21 November 2001

\section{References}

1 Scott JA, Clearkin LG. Surgically induced scleritis following cataract extraction. Eye 1994:8:292-7.

2 Desai P, Minassian DC, Reidy A. National cataract surgery survey 1997-8; a report of the results of the clinical outcomes. $\mathrm{Br} J$ Ophthalmol 1999;83:1336-40.

3 Sainz-de-la Maza M, Jabbur NS, Foster CS. An analysis of therapeutic decision for scleritis. Ophthalmology 1993;100:13726.Br J Ophthalmol

\section{Total exudative detachment as a} first presentation of von Hippel

\section{Lindau disease}

Von Hippel Lindau disease is a rare condition characterised by retinal and central nervous system haemangioblastomas. It is also associated with renal cell carcinoma, phaeochromocytoma, and renal, pancreatic, and epididymal cysts.

The disease usually presents with neurological symptoms and/or visual disturbance angiomas if seen in the retina can often be treated in an attempt to prevent progression to retinal detachment.

We report a young girl with von Hippel Lindau disease whose initial presentation was with a total exudative retinal detachment.

\section{Case report}

A 14 year old girl presented to the eye casualty department with no perception of light in her left eye. She gave a 2 month history of gradual, painless loss of vision, but had delayed previously mentioning her symptoms.

Past ocular history was unremarkable; an optometrist visit a year previously was normal. She was otherwise well.

Slit lamp examination on the left revealed a quiet anterior segment, with retinal folds visible abutting the posterior lens capsule (Fig 1A). The right eye was entirely normal. An ultrasound B-scan showed a left total funnel retinal detachment (Fig 1B). Computed tomograph scan demonstrated normal orbits.

The patient's identical twin was also present and was examined: both her eyes were normal.

At this stage the family history proved helpful. The patient's father had recently been diagnosed with renal cell carcinoma and pituitary vascular tumours.

The paternal line had a cluster of various carcinomas, including three relatives with cerebellar tumours. Further investigation revealed at least one of these to be histologically 

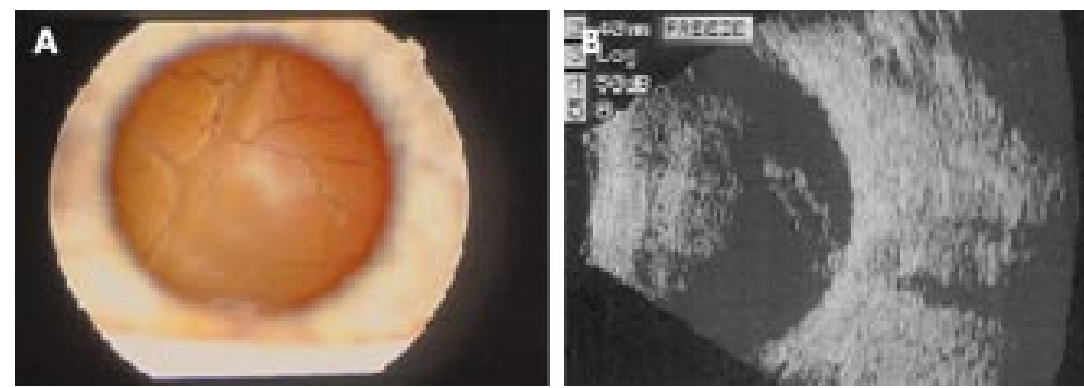

Figure 1 (A) Retinal folds visible behind lens. (B) Ultrasound B-scan showing funnel retinal detachment.

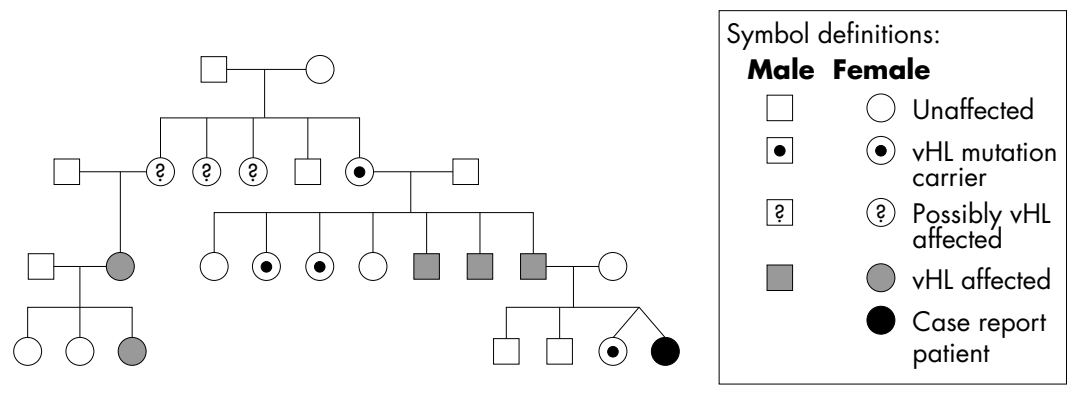

Figure 2 Family pedigree.

confirmed haemangioblastoma. The possibility of von Hippel Lindau disease was raised and the patient's father was screened: the diagnosis was confirmed with polymerase chain reaction analysis; showing a frame shift mutation (single base pair deletion [G]) in codon 195 (exon 3) of the von Hippel Lindau gene. Subsequent extended family screening has revealed the same mutation in the patien and nine other living relatives (Fig 2), including the patient's identical twin. Five of these relatives have clinical manifestations of the disease, and in one case an asymptomatic stage 1 renal cell carcinoma was diagnosed and successfully resected.

Miss AB's left eye has subsequently developed pupil block glaucoma which is currently well controlled medically. It remains blind with a rigid total retinal detachment. The cause of this detachment is most likely to be an optic nerve or retinal haemangioblastoma; however, no causal lesion was identified on ultrasound or computed tomography (CT). The few retinal vessels that can be seen (Fig 1A) do not show any abnormality. Her other eye remains normal and screening for signs of the disease elsewhere has proved negative.

\section{Comment}

This case highlights the importance of considering von Hippel Lindau disease as a diagnosis when confronted with a patient with an unexplained exudative retinal detachment.

Von Hippel Lindau disease has an autosomal dominant inheritance pattern with an incidence of one in 36000 ; with a $70 \%$ prevalence of ocular haemangioblastomas. Von Hippel Lindau gene mutation carriers have a $35 \%$ probability of visual loss by age 50 .

Ocular angiomas occur commonly in the superotemporal mid-peripheral retina, less commonly on the optic disc $(8 \%)$, and at the posterior pole $(1 \%)$.

If left untreated, lesions can cause vitreous haemorrhage, macular oedema, epiretinal membrane formation, and exudative or tractional retinal detachment. This last complication is well demonstrated in our patient's case, with a 2 month delay between onset of symptoms and first presentation.

Treatment comprises laser photocoagulation of angiomas less than one disc diameter. Cryotherapy, or vitreoretinal surgery with trans-scleral drainage and endolaser may be indicated in larger lesions, ${ }^{3}$ especially if vitreous traction is present. ${ }^{2}$ Early treatment necessarily offers a better prognosis.

This case underscores the value of taking a good family history in order to detect hereditary diseases, which can then be confirmed with genetic molecular analysis. The importance of identification and subsequent screening of von Hippel Lindau gene mutation carriers (both affected and unaffected) is exemplified in our patient's family - with the potential benefit of early diagnosis and treatment of asymptomatic retinal and cerebellar haemangioblastomas, as well as potentially fatal tumours. The screening should be maintained regularly, as the protean manifestations of von Hippel Lindau disease may occur de novo at any age.

A Ferguson, J Singh Princess Alexandra Eye Pavilion, Chalmers Street, Edinburgh EH3 9HA, UK

Correspondence to: Andrew Ferguson aferguson@doctors.org.uk

Accepted for publication 9 January 2002

\section{References}

1 Webster AR, Maher ER, Moore AT. Clinical characteristics of ocular angiomatosis in von Hippel-Lindau disease and correlation with germline mutation. Arch Ophthalmol 1999; 117:371-8. angiomatosis: the ocular manifestations of von Hippel-Lindau disease. Can J Ophthalmol 1986;21:276-83.

3 Garcia-Arumi J, Sararols LH, Cavero L, et al. Therapeutic options for capillary papillary hemangiomas. Ophthalmology
2 Ridley M, Green J, Johnson G. Retinal 2000; 107:48-54
Anomalous venous drainage of a plexiform (pial) arteriovenous malformation mimicking an indirect caroticocavernous sinus fistula

We report a case of a 35 year old man who presented with proptosis of the left globe and congested episcleral and retinal veins. We present angiographic evidence to show that the venous engorgement of the left orbit was related to anomalous venous drainage of a previously posteriorly draining parietal arteriovenous malformation (AVM).

\section{Case report}

A 35 year old man presented complaining of a 3 month history of a red eye and a 1 month history of progressive swelling and protrusion of the left eye. His only significant past medical history was that of a subarachnoid haemorrhage from an intracranial AVM 7 years previously for which he had undergone stereotactic radiosurgery. Ocular examination revealed a visual acuity of $6 / 5$ in either eye. There was a $5 \mathrm{~mm}$ axial displacement of the left globe with normal ocular motility. The proptosis was non-pulsatile, non-reducible, and Valsalva manoeuvre was negative. There was no audible bruit. Anterior segment examination was unremarkable aside from congested episcleral vessels. Examination of the fundus revealed dilated and tortuous retinal veins but a normal arterial system. There was no disc swelling.

A computed tomography (CT) scan of the brain and orbits revealed an AVM in the left primary sensory cortex, proptosis of the left globe and a dilated superior ophthalmic vein (Fig 1). A magnetic resonance image (MRI) with angiography confirmed the presence of an AVM in the left perirolandic region which was fed by the left middle cerebral artery and the pericallosal branch of the left anterior cerebral artery (Fig 2). The dominant venous drainage was via a large superficial vein upwards to the superior sagittal sinus. Although the AVM did not appear to have changed significantly in overall size when compared with previous angiograms (Fig 3), it was clear that there had been significant changes in the venous structures distant to the AVM. A relative constriction at the junction of the ipsilateral transverse and sigmoid sinuses had developed (Fig 4) and as

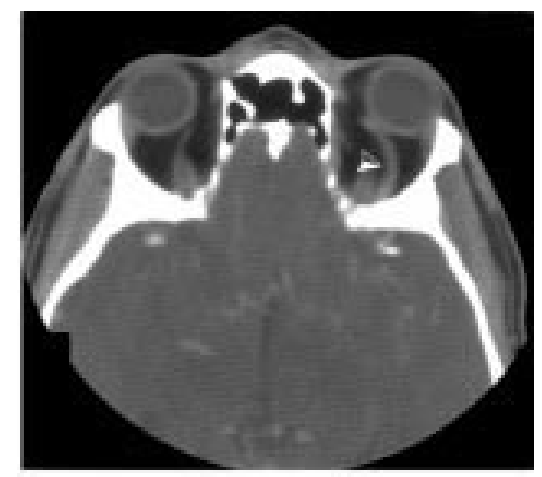

Dilated superior ophrhalmic vein

Figure 1 CT scan of the orbits showing dilated superior ophthalmic vein. The majority of the venous outflow from the cavernous sinus is via the superior ophthalmic vein which is markedly dilated as seen in this scan. 

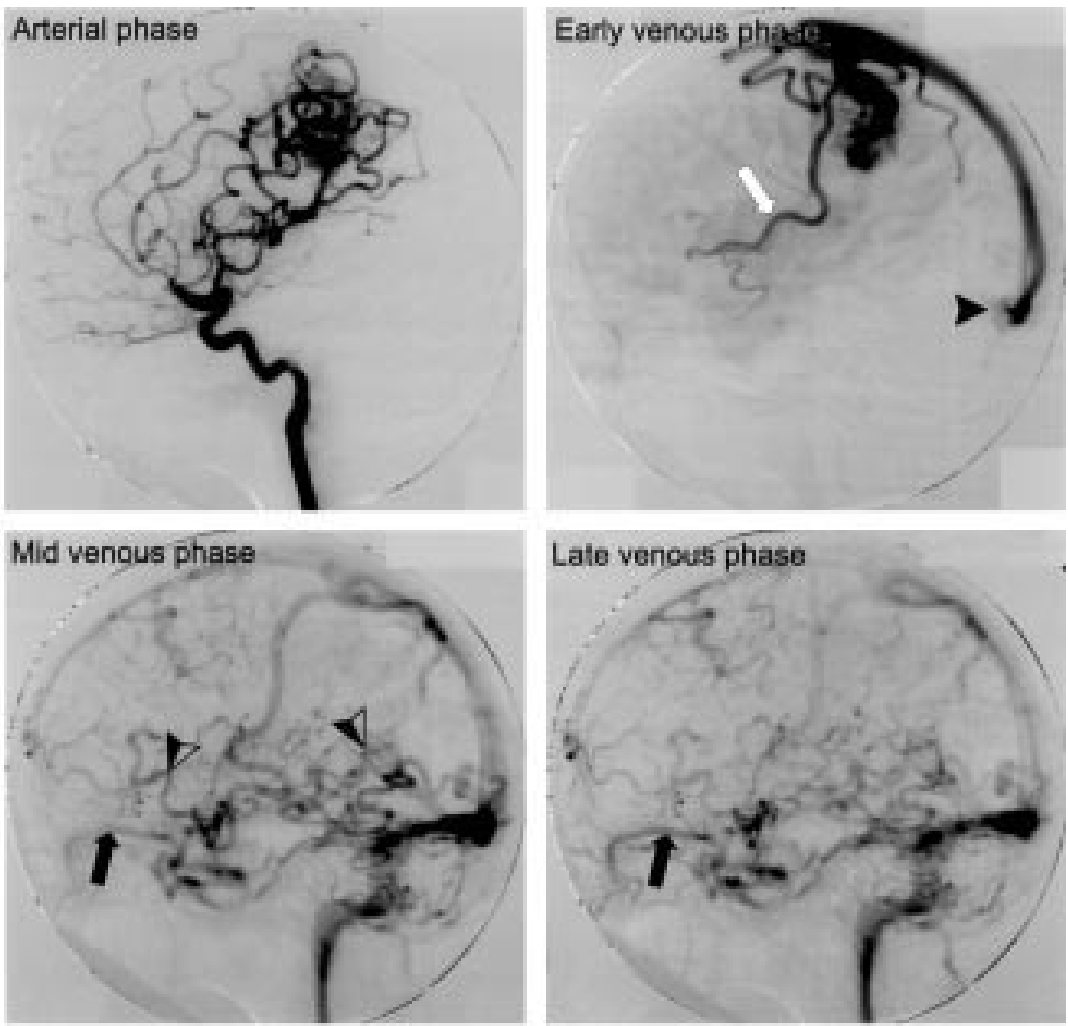

Relatively restricted flow into sigmoid sinus doe to stenosis at transverse' sigmoid sinus junction.

Increased diameler of cortical dnining veins. $\Longrightarrow$

Dilated superior ophthalmis vein.

Multiple newly opened collmeral venous chanels $D$

Figure 2 Anterior segment photographs showing the dilated episcleral vessels in the left eye.

a consequence there was a relative hold up to the filling of the sigmoid sinus. The AVM therefore now drained anteriorly via a collateral circulation into the cavernous sinus. The later phases of the angiogram revealed that there was relatively little downward venous outflow from the cavernous sinus and the majority of this venous outflow was therefore shunted via the superior ophthalmic vein

which was markedly dilated as a consequence. The patient's symptoms of unilateral proptosis and venous engorgement were therefore a manifestation of increased blood flow through collateral shunts that had developed as a consequence of a stricture in the principal drainage channel of the existing AVM, which itself had not changed significantly in size.
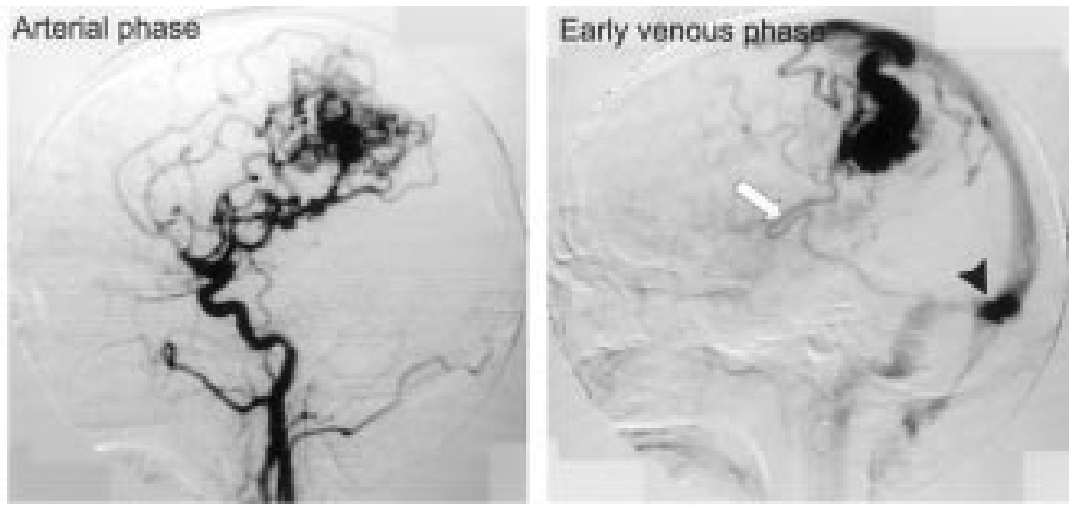

Normal (non restricted) flow through transwerse simus.

\section{Small cortical draining vessels. $\Longrightarrow$}

Figure 3 Cerebral angiogram dated 1994. A moderately sized arteriovenous malformation is shown fed by branches of the left middle cerebral and anterior cerebral arteries. It is drained by a large superficial vein which continues into the superior sagittal sinus.

\section{Comment}

Arteriovenous malformations (AVM) are lesions which consist of racemose networks of arterial and venous channels which communicate directly rather than through a normal capillary bed. These communications are of two types: fistulous and plexiform. In the fistulous type an arterial channel empties directly into a venous channel, while in the plexiform type one or more arterial channels feed a vascular conglomerate that comprises multiple arteriovenous communications from which one or more venous channels emerge as draining veins. ${ }^{1}$ The fistulous types are usually supplied by meningeal branches of the external carotid artery and therefore they are also known as dural AVMs. The plexiform type, in contrast, are supplied by branches of the cerebral or cerebellar arteries and hence are also known as pial AVMs. The AVM most commonly encountered by the ophthalmologist is the acquired caroticocavernous sinus fistula, a dural AVM whose characteristic neuroophthalmic presentation results from the arterialisation of the orbital venous system. Proptosis and orbital venous engorgement secondary to direct arterialisation of the venous system has also been reported in dural AVMs involving the torcular herophili ${ }^{2}$ and the Galenic system. ${ }^{3}$ The more common plexiform (pial) AVMs typically present with complications that arise from the massive venous run off that is generated by the associated arteriovenous shunts-namely, intracranial haemorrhage, seizures, and recurrent headache. ${ }^{4}$ Symptomatic orbital drainage of plexiform (pial) AVMs is rare and when it does occur the AVM is usually in an anterior location..$^{-58}$

We describe a patient with a posteriorly located plexiform (pial) AVM who presented with proptosis and vascular engorgement of the left globe as a result of the venous outflow of the AVM being shunted anteriorly through newly opened collateral channels. Unilateral proptosis associated with anterior shunting has previously been described in a child with bilateral sigmoid sinus hypoplasia, ${ }^{9}$ but has never been described in a patient with a posteriorly located plexiform AVM. Our case is also extremely unusual in that we have angiographic evidence which shows that these collateral channels developed as a result of an acquired stenosis at the junction of the transverse and sigmoid sinus, previously its dominant route of venous drainage. The aetiology of this focal stenosis is unknown. It is unlikely to be a consequence of the stereotactic radiosurgery as the site of the stenosis is remote to the previously treated area. It is more likely that the stenosis represents a response to the chronic endothelial damage that is known to accompany the hyperdynamic circulation of AVMs. ${ }^{1}$

This case serves to illustrate that not all adults who present with proptosis and venous engorgement of the globe have a caroticocavernous sinus fistula and other anomalies of venous drainage must occasionally be considered.

D Squirrell, P Puri, P A Rundle

Department of Ophthalmology, Royal Hallamshire Hospital, Sheffield S10 2JF, UK

C Romanowski

Department of Radiology

I G Rennie

University Department of Ophthalmology and Orthoptics, Floor O, Royal Hallamshire Hospital, Sheffield S10 2RX, UK

Correspondence to: Mr P Puri

Accepted for publication 4 February 2002 

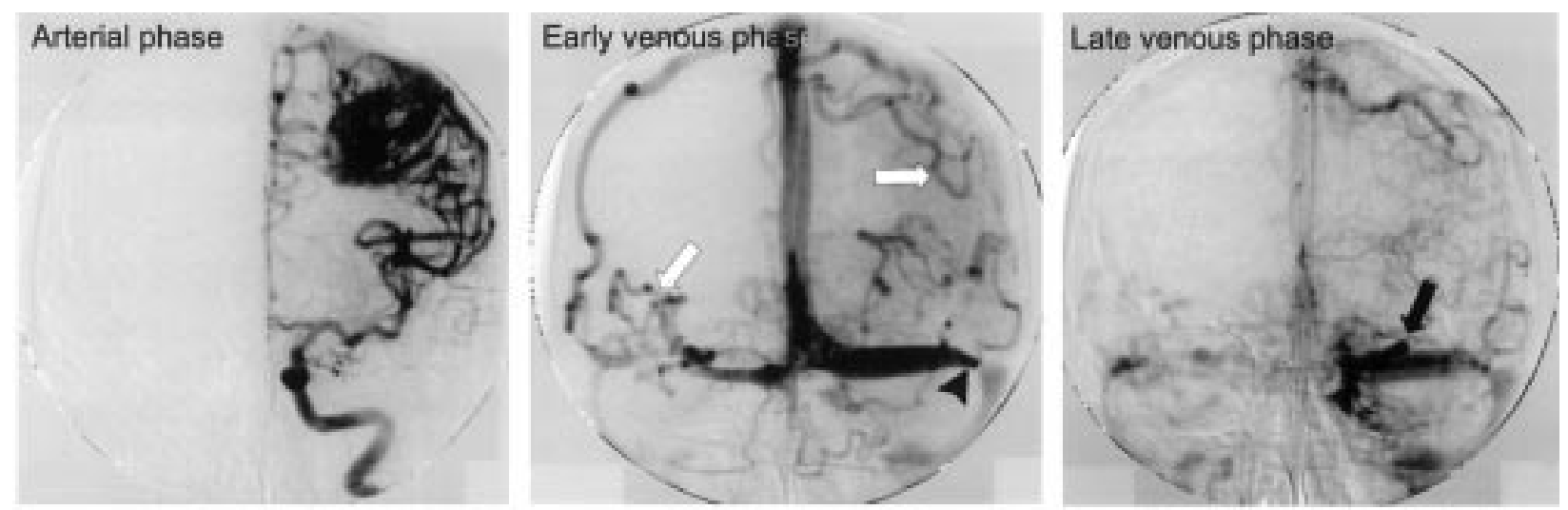

Stenosis at the junction of the transverse and sigmoid sinus.

Multiple newly opened collateral venous channels. $\Rightarrow$

Dilated superior ophthalmic vein.

Figure 4 Cerebral angiogram dated 2000. There has been no significant change in the size of the arteriovenous malformation since the angiogram was performed in 1994. There is constriction at the junction of the transverse and sigmoid sinuses with hold up in the transverse sinus. Multiple cortical collateral vessels are seen draining into the cavernous sinus.

\section{References}

1 Valavanis A. The role of angiography in the evaluation of cerebral vascular malformations. Neuroimaging Clin N Am 1996;6: 679-704.

2 Buchnanan TA, Harper DG, Hoył WF. Bilateral proptosis, dilation of conjunctival veins and papilloedema: a neuro-ophthalmological syndrome caused by arteriovenous malformation of the torcular herophili. Br J Ophthalmol 1982;66: 186-9.

3 Eckman PB, Fountain EM. Unilateral proptosis. Association with arteriovenous malformations involving the galenic system. Arch Neurol 1974:31:350-1.

4 Mackenzie I. The clinical presentation of the cerebral angioma; a review of 50 cases. Brain 1953;76:184-214.

5 Sibony PA, Lessell S, Wray S. Chiasmal syndrome caused by arteriovenous malformations. Arch Ophthalmol 1982;100:438-42

6 Volpe NJ, Sharma MC, Galetta SL, et al. Orbital drainage from cerebral arteriovenous malformations. Neurosurgery 2000;46:820-4.

7 Malzone WF, Gonyea EF. Exophthalmos with intracerebral arteriovenous malformations. Neurology 1973;23:534-8.

8 Forman AR, Luessenhop AJ, Limaye SR. Ocular findings in patients with arteriovenous malformations of the head and neck. Am J Ophthalmol 1975;79:626-33.

9 Tech KE, Becker CJ, Lazo A, et al. Anomalous Intracranial venous drainage mimicking orbital or cavernous arteriovenous fistula. $\mathrm{Am} \mathrm{J}$ Neuroradiol 1995;16:171-4

\section{Childhood myasthenia gravis in an infant}

Myasthenia gravis is defined as an acquired autoimmune disorder where there is abnormal fatiguability of muscles due to deficiency of acetylcholine receptors caused by circulating antibodies directed against them. ${ }^{1}$ Ocular myasthenia is a form of myasthenia gravis clinically involving only the levator palpebrae superioris, the orbicularis oculi, and the extraocular muscles. Ptosis and ophthalmoplegia, both unilateral and bilateral, constitute the only signs in about $20 \%$ cases while in about $70 \%$ cases, ocular symptoms mark the onset of generalised myasthenia gravis.

\section{Case report}

We examined a 10 month old male child who presented to us with complaints of ptosis and ophthalmoplegia of the right eye for a month before presentation. The child was born normally at full term to a healthy young mother with an uneventful perinatal and postnatal period. The milestones of the child were recorded as normal. The child had an older sister aged 3 years who gave no history of similar complaints. There was no history of fever, vomiting, pain, trauma, seizures, difficulty in swallowing or chewing, or any history of excessive lassitude or listlessness given by the parents with regard to the child.

General physical examination revealed a child with normal physical and mental development. Systemic examination revealed no presence of heart block or any other cardiovascular abnormality. Neurological examination revealed no motor weakness, incoordina-

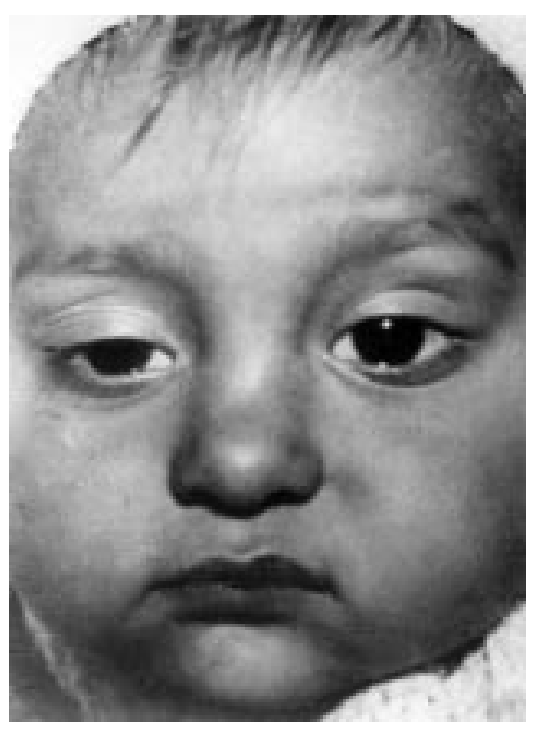

Figure 1 Clinical photograph of the patient showing ptosis of the right upper lid with ophthalmoplegia. There is no strabismus present in the primary gaze position. tion, ataxia, or areflexia. On ocular examination, the patient had moderate ptosis of the right upper lid with absence of any voluntary movement of the right eyeball. Ocular movements of the left eye appear to be normal. The child was attentive to both visual and auditory stimuli and frequently changed his head posture to look in the direction of origin of the visual or auditory stimuli. The pupillary reactions were normal in both eyes. In the primary gaze position, the visual axes of both eyes were parallel (Fig 1). The child was examined twice after a period of sleep and similar findings were recorded again.

Examination of the anterior and posterior segment of both eyes under general anaesthesia was unremarkable. The forced duction test was negative. Based on the above findings, a tentative diagnosis of an intracranial space occupying lesion was made. However, a contrast enhanced computed tomograph (CT) scan of the head and orbit revealed no abnormalities. Cerebrospinal fluid examination was also normal.

The child was subsequently subjected to an edrophonium test $(0.15 \mathrm{mg} / \mathrm{kg}$ body weight intravenously), which was unequivocally positive with complete resolution of the ptosis and ophthalmoplegia (Fig 2). A repetitive stimulation of the left median nerve demonstrated a significant decremental response $(22 \%)$. Subsequently, a serum analysis of antiacetylcholine receptor binding, blocking, and modulating antibodies was performed. Abnormal titres of anti-acetylcholine receptor binding antibodies $(5.8 \mathrm{nmol} / \mathrm{l}$; normal is less than $0.8 \mathrm{nmol} / \mathrm{l}$ ) were present which confirmed the diagnosis of childhood myasthenia gravis. A contrast enhanced CT scan of the thorax was normal. The child was started on tablet neostigmine $3 \mathrm{mg}$ once daily along with oral prednisolone $(0.5 \mathrm{mg} / \mathrm{kg}$ body weight) and followed up regularly. Marked improvement of the ptosis and ophthalmoplegia was observed which persisted at 1 year of follow up. Systemic steroids were gradually tapered off after 6 months.

\section{Comment}

Our patient was diagnosed to have juvenile myasthenia gravis, which comprises approximately $1 \%$ of all cases of myasthenia gravis. ${ }^{2}$ It 


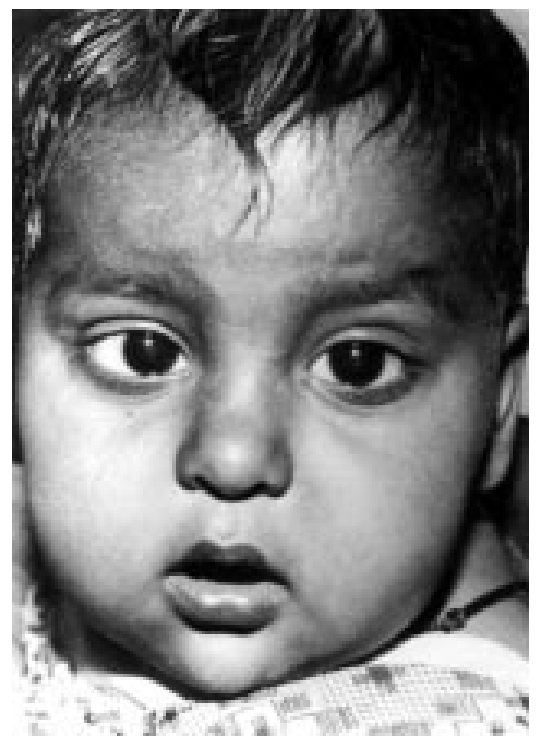

Figure 2 Clinical photograph of the same patient showing marked improvement of the ptosis and ophthalmoplegia after administration of intravenous edrophonium.

is considered to be a variant of adult myasthenia with similar clinical and autoimmune mechanism of production. ${ }^{3}$ In contrast with congenital myasthenia gravis which occurs more commonly in children less than 2 years of age, has a familial mode of occurrence, and is not responsive to steroid therapy, ${ }^{3}{ }^{4}$ juvenile myasthenia gravis presents usually after 5 years of age, shows no familial occurrence, and is characterised by a definite autoimmune mechanism. ${ }^{34}$ Though cases of juvenile myasthenia gravis with age of onset at 2 years or less ${ }^{35}$ have been reported, to the best of our knowledge our patient is the youngest patient with the above diagnosis reported so far. We would like to reiterate that investigations to rule out myasthenia gravis should be performed in all patients with acquired ptosis and ophthalmoplegia, irrespective of age.

Z Chaudhuri, P K Pandey, S Bhomai, D Chauhan, L U Rani

Department of Ophthalmology, Guru Nanak Eye Centre, New Delhi - 110002, India

Correspondence to: Dr Zia Chaudhuri, E-310,

Purvasha, Anand Lok Society, Mayur Vihar phase 1, Delhi-1 10091, India; drzia@bol.net.in

Accepted for publication 20 January 2002

No grants or sponsorships have been requisitioned for this study. The authors do not have any proprietary or financial interest in any product or procedure mentioned in the manuscript.

\section{References}

1 Weinberg DA, Lesser RL, Vollmer TL. Ocular myasthenia: a protean disorder. Surv Ophthalmol 1994;39:169-209.

2 Kini PG. Juvenile myasthenia gravis with predominant facial weakness in a 7-year-old boy. Int J Pediatr Otorhinolaryngol 1995;32:167-9

3 Jaison SG, Abraham AP. Childhood myasthenia gravis in a toddler. Ind J Ophthalmol 1995:43:136-7.

4 Millichap JG, Dodge PR. Diagnosis and treatment of myasthenia gravis in infancy, childhood and adolescence. Neurology 1960;11:1007-14

5 Verma AK, Behari M, Ahuja GK. Myasthenia gravis in the young. Indian J Pediatr 1986;23:363-9

\section{Phototoxic maculopathy} following uneventful cataract surgery in a predisposed patient

Operating microscope light induced foveal damage is a well recognised occurrence following ocular surgery including complicated cataract extraction, complex anterior segment procedures, and vitrectomy surgery. An increased risk of phototoxicity is associated with an operating time greater than 100 minutes, increased body and therefore retinal temperature, unfiltered blue light, and hyperoxaemia. ${ }^{23}$

We report a patient who developed a phototoxic lesion during routine cataract surgery possibly related to underlying systemic lupus erythematosus (SLE) and hydroxychloroquine treatment. We examine the measures taken to prevent recurrence with second eye surgery and the implications for routine cataract surgery are discussed.

\section{Case report}

A 39 year old woman presented with blurred left vision and glare. One year previously she had been diagnosed with SLE for which she had been taking hydroxychloroquine $200 \mathrm{mg}$ and prednisolone $5 \mathrm{mg}$ daily for 18 months. She had bilateral subcapsular cataracts and underwent routine left phacoemulsification and lens implant surgery under general anaesthesia.

Directly after surgery, the patient noted two confluent blind spots immediately below fixation in the left eye. When first seen by us the left visual acuity was $6 / 5$, intraocular pressure was $16 \mathrm{~mm} \mathrm{Hg}$, and the eye was quiet with a well centred posterior chamber lens implant. Fundal examination of the left eye showed two well circumscribed areas of coarse mottled retinal pigment epithelial (RPE) disturbance, approximately one and a half disc diameters in size, superotemporal to the fovea (Fig 1). There was no subretinal fluid or haemorrhage associated with these lesions. A fluorescein angiogram supported these findings (Fig 2) and the lesions were felt to be consistent with photic injury.

The patient was keen to proceed with right phacoemulsification and lens implantation in view of anisometropia. She had stopped her hydroxychloroquine immediately after the first operation and the following precautions were agreed with the patient before surgery in the second eye: the use of the microscope filter throughout surgery, switching off the microscope light when instruments were not in the eye, tilting of the eye into downgaze (so that any phototoxic lesion would be superior to fixation and not result in a homonymous scotoma), and the maintenance of low body

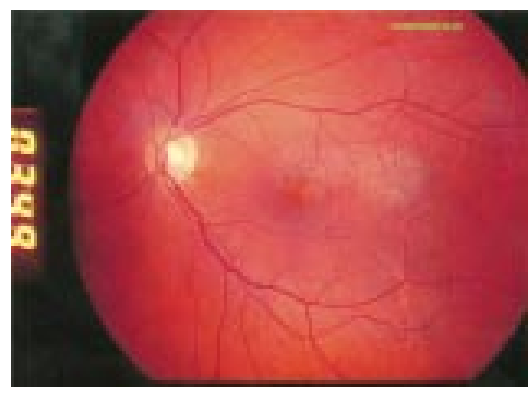

Figure 1 Left eye showing two well circumscribed areas of coarse mottled retinal pigment epithelial disturbance.

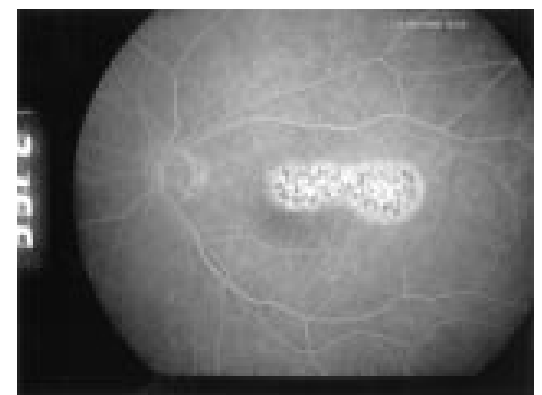

Figure 2 Fluorescein angiogram of left eye.

temperature and low inspired oxygen concentration during surgery.

An uncomplicated right phacoemulsification and lens implant was subsequently performed. Two weeks after surgery, the right visual acuity was $6 / 5$, anisometropia was abolished, and funduscopy was normal.

\section{Comment}

Phototoxic lesions are defined as the retinal lesions produced after a relatively short exposure to an intense light source such as the operating microscope. ${ }^{1}$ Historically, these lesions were typically located inferior to the fovea as a result of the slight downgaze of the eye during extracapsular cataract surgery and a coaxial microscope beam directed inferior to the fovea. ${ }^{134}$ The incidence of retinal phototoxicity from the operating microscope has been reported at between $0 \%$ and $28 \%$, with large series reporting angiographic evidence of retinal phototoxicity in $3-7 \%$ of

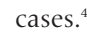

Retinal phototoxic lesions first appear as well circumscribed outer retinal whitening (oedema) with mild disturbances of the RPE often with a light border visible after a few days. ${ }^{4}$ Ophthalmoscopically, a subtle discrete margin can be seen. After the first week, lesions are characterised by coarse alterations of the RPE layer with fluorescein angiography demonstrating characteristic early discrete mottled hyperfluorescence with late staining but no leakage. ${ }^{4}$

Certain photosensitising drugs, such as hydroxychloroquine, have been reported to predispose to the development of retinal phototoxic lesions. However, there are no published reports of a similar association with systemic photosensitive conditions such as SLE. It has been suggested that patients who have SLE have increased numbers of chromosome breaks and rearrangements correlated with a low molecular weight chromosome damaging agent (clastogenic factor) present in lymphocytes that sensitises them to near ultraviolet light (360-400 nm) light. ${ }^{5}$ The presence of this photoactivated agent explains why SLE patients show an aggravation of their characteristic skin condition after exposure to sunlight. Certain pharmacological agents, such as hydroxychloroquine, which is used in the treatment of SLE, can also predispose to the development of phototoxicity. The mechanisms behind the predisposition to phototoxicity in humans of chloroquine and its derivatives are less clear. However, it is known that these drugs accumulate in the RPE layer of the retina and can cause drug induced lysosomal abnormalities including diminished vesicle fusion, diminished exocytosis, and reversible "lysosomal storage disease." ${ }^{\prime \prime}$ These two different predisposing mechanisms to phototoxicity presumably coexisted in this patient and may have significantly increased her risk. 
Several procedures were undertaken to prevent the occurrence of a phototoxic lesion in the second eye. These included the use of ultraviolet filters on the microscope, although air can also be used in the anterior chamber to defocus the light from the retina as can light barriers on the cornea or in the microscope. The time and power of coaxial illumination from the microscope was minimised by turning the microscope off when instruments were not in the eye, and the patient's eye was kept in downgaze so that the operating microscope's axis was aligned off the patient's visual axis so that any phototoxic lesion would be extrafoveal and no cause a homonymous scotoma. Since anima studies have demonstrated a benefit of altering core ocular temperature, ${ }^{13}$ the patient's body temperature was kept low to reduce the temperature within the eye. Alternatively, irrigation solutions may be cooled relative to room temperature to help reduce intraocular temperature. It has been shown that an increase in inspired oxygen markedly en hances retinal phototoxicity and so a low inspired oxygen concentration was used for the patient during the procedure. ${ }^{13}$ The patient discontinued hydroxychloroquine treatment following the surgery to her left eye but ophthalmologists should be aware of the risk of phototoxic retinal lesions in patients taking photosensitising pharmacological agents particularly for underlying potential photosensitising systemic conditions. Consideration should be given to stopping treatment before surgery and taking appropriate surgical precautions.

B Manzouri, C A Egan, P G Hykin Medical Retina Service, Moorfields Eye Hospital City Road, London EC1V 2PD, UK

Correspondence to: Mr P G Hykin phil.hykin@moorfields.nhs.uk

Accepted for publication 11 February 2002

\section{References}

1 Michels M, Sternberg P. Operating microscope-induced retinal phototoxicity: pathophysiology, clinical manifestations and prevention. Surv Ophthalmol 1990;34:237-52.

2 Stamler JF, Blodi CF, Verdier D, et al. Microscope light-induced maculopathy in combined penetrating keratoplasty, extracapsular cataract extraction, and intraocular lens implantation. Ophthalmology 1988;95:1142-6.

3 Davidson PC, Sternberg P. Potential retinal phototoxicity. Am J Ophthalmol 1993:116:497-501.

4 Michels $M$. Intraoperative retinal phototoxicity. Int Ophthalmol Clin 1995;35: 157-72.

5 Emerit I, Michelson AM. Mechanism of photosensitivity in systemic lupus erythematosus patients. Proc Natl Acad Sci USA 1981;78:2537-40.

6 Motten AG, Martinez LF, Holt N, et al. Photophysical studies on antimalarial drugs.

Photochem Photobiol 1999:69:282-7.

\section{Bilateral uveitis after intravesical BCG immunotherapy for bladder carcinoma}

Intravesical bacille Calmette-Guerin (BCG) is indicated for the treatment and prophylaxis of carcinoma in situ of the urinary bladder and prophylaxis of primary or recurrent papillary tumours. ${ }^{1}$ All previous reports of uveitis after intravesical BCG therapy have been associated with arthritis. ${ }^{1-4}$ HLA-B27 has been positive in all cases with uveitis. ${ }^{1-4}$ We describe a patient who is HLA-B27 negative with isolated bilateral uveitis after intravesical BCG therapy.

\section{Case report}

A 70 year old man presented to the eye casualty department with a 4 day history of bilateral red, painful, and photophobic eyes. He had no systemic complaints. He had no ophthalmic history of note. He had a medical history of hypertension, gout, and recurrent papillary transitional cell carcinoma of the bladder. Eight weeks previously he had been seen by a urologist for haematuria and increased frequency. A diagnosis of recurrent papillary cell carcinoma was made by cystoscopy and biopsy. A 6 week course of weekly intravesical BCG therapy was commenced. His medications on presentation were Adala (nifedipine) and amlodipine. He was allergic to penicillin and aspirin.

On ocular examination his visual acuities were 6/12 right eye and 6/6 left eye. A diagnosis was made of bilateral acute anterior uveitis. He had $2+$ cells and posterior synechiae bilaterally. No keratic precipitates were seen. He had a right sided epiretinal membrane.

Laboratory studies showed an erythrocyte sedimentation rate of 66 with the rest of haematological studies normal. Biochemistry showed a raised sodium, urea, and creatinine, which were longstanding. Tests for antinuclear antibodies and rheumatoid factor were negative. Angiotensin converting enzyme was normal and Treponema pallidum antibodies were not detected. HLA-B27 was negative. Chest and pelvis radiographs were normal. His uveitis resolved completely on topical steroid therapy and mydriatics. There was no recurrence of his uveitis over the subsequent follow up period of 3 months.

\section{Comment}

There have been no reported cases of intravesical BCG causing isolated uveitis in a patient that is HLA-B27 negative. There have been 26 reported cases of reactive arthritis secondary to intravesical BCG. ${ }^{2}$ About $8 \%$ of these cases were associated with uveitis. ${ }^{2}$ Al the reported cases of combined arthritis and uveitis were HLA-B27 positive. ${ }^{134}$ In the BCG related arthritides the mean time of presentation was 10.5 days after the last BCG therapy. ${ }^{2}$ Our patient started having ocular symptoms 10 days after his last BCG therapy.

In a review of 1278 patients treated with intravesical BCG for bladder cancer, $95 \%$ had no complications. ${ }^{5}$ There were no reported cases of uveitis. Arthritis and arthralgia accounted for $0.5 \%$ of the complications. ${ }^{5}$

There has been one reported case of endophthalmitis from Mycobacterium bovisBCG used to treat bladder cancer. ${ }^{6}$ This was proved microbiologically and histologically. This presentation was delayed with the patient presenting 14 months after his last intravesical BCG therapy.

Intravesical BCG contains live attenuated mycobacteria. The intravesical BCG promotes a local acute inflammatory and subacute granulomatous reaction with macrophage and lymphocyte infiltrates in the superficial bladder. ${ }^{7}$ The exact mechanism of action is unknown but the antineoplastic effect seems to be $\mathrm{T}$ lymphocyte dependent. ${ }^{7}$ This enhanced cell mediated immunity results in recognition and suppression of neoplastic cells.

It has been hypothesised that the systemic inflammatory response is seen as part of an immune response initiated by BCG and then continued by cross reactivity with particular host proteins presented by the class I MHC receptor. ${ }^{9}$
It is impossible to definitively prove that intravesicular BCG caused our patient's bilateral anterior uveitis. The possibility of an infective aetiology by active mycobacterium must also be considered. If this were the case definitive diagnosis could have been established by sending a specimen of aqueous for acid fast staining, culture, and polymerase chain reaction (PCR)

It is plausible that intravesicular BCG can cause acute inflammatory and hypersensitivity reactions in the eye secondary to a systemic inflammatory response. ${ }^{1-5}$ Contrary to previous thought, HLA-B27 need not be a determining factor for uveitis associated with intravesicular BCG. ${ }^{3}$ This is the first reported case of isolated bilateral anterior uveitis presumed secondary to intravesicular BCG in a patient who is HLA-B27 negative.

M Wertheim, N Astbury

Eye Department, Norfolk and Norwich University Hospital, Colney Lane, Norwich NR4 7UZ, UK

Accepted for publication 13 February 2002

\section{References}

1 Price GE. Arthritis and iritis after BCG therapy for bladder cancer. J Rheumatol 1994:21:564-5.

2 Clavel G, Grados F, Cayrolle G, et al. Polyarthritis following intravesical BCG immunotherapy. Rev Rheum Engl Ed 1999;66:115-18

3 Chevrel G, Zech C, Miossec P. Severe uveitis followed by reactive arthritis after bacillus Calmette-Guerin therapy. J Rheumatol 1999;26:1011.

4 Missioux D, Hermabessiere J, Sauvezie B. Arthritis and iritis after bacillus Calmette-Guerin therapy. J Rheumatol 1995;22:2010.

5 Lamm DL, Stodgdill VD, Stodgill B, et al. Complications of bacillus Calmette-Guerin immunotherapy in 1,278 patients with bladder cancer. J Urol 1986;135:272-4.

6 Han DP, Simons KB, Tarkanian CN, et al. Endophthalmitis from Mycobacterium bovis-bacille Calmette-Guerin after intravesicular bacilli Calmette-Guerin injections for bladder cancer. Am J Ophthalmol 1999:128:648-50.

7 O'Donnell MA, DeWolf WC. BCG immunotherapy for superficial bladder cancer New prospects for an old warhorse. Surg Oncol Clin North Am 1995:4:189-202.

8 Sokal JE, Aungst CW, Han T. Effect of BCG on delayed hypersensitivity responses of patients with neoplastic disease. Int J Cancer 1973;12:242-9.

9 Puett DW, Fuchs HA. Arthritis after bacillus Calmette-Guerin therapy. Ann Intern Med 1992; 117:537.

\section{NOTICES}

\section{Childhood blindness}

The latest issue of Community Eye Health (No 40 discusses new issues in childhood blindness, with an editorial by Clare Gilbert, senior lecturer at the International Centre for Eye Health. For further information please contact: Journal of Community Eye Health, International Centre for Eye Health, Institute of Ophthalmology, 11-43 Bath Street, London ECIV 9EL, UK (tel: +44 (0)20 7608 6910; fax: +44 (0)20 7250 3207; email: eyeresource@ucl.ac.uk; website: www.jceh.co.uk). Annual subscription (4 issues) UK£25/US\$40. Free to workers in developing countries.

\section{International Centre for Eye Health}

The International Centre for Eye Health has published a new edition of the Standard List of 
Medicines, Equipment, Instruments and Optical Supplies (2001) for eye care services in developing countries. It is compiled by the Task Force of the International Agengy for the Prevention of Blindness. Further details: Sue Stevens, International Centre for Eye Health, 11-43 Bath Street, London ECIV 9EL, UK (tel: +44 (0)20 7608 6910; email: eyeresource@ucl.ac.uk).

\section{Second Sight}

Second Sight, a UK based charity whose aims are to eliminate the backlog of cataract blind in India by the year 2020 and to establish strong links between Indian and British ophthalmologists, is regularly sending volunteer surgeons to India. Details can be found at the charity website (www.secondsight.org.uk) or by contacting Dr Lucy Mathen (lucymathen@yahoo.com).

\section{SPecific Eye ConditionS (SPECS)}

SPecific Eye ConditionS (SPECS) is a not for profit organisation which acts as an umbrella organisation for support groups of any conditions or syndrome with an integral eye disorder. SPECS represents over fifty different organisations related to eye disorders ranging from conditions that are relatively common to very rare syndromes. We also include groups who offer support of a more general nature to visually impaired and blind people. Support groups meet regularly in the Boardroom at Moorfields Eye Hospital to offer support to each other, share experiences and explore new ways of working together. The web site www.eyeconditions.org.uk acts as a portal giving direct access to support groups own sites. The SPECS web page is a valuable resource for professionals and may also be of interest to people with a visual impairment or who are blind. For further details about SPECS contact: Kay Parkinson, SPECS Development Officer (tel: +44 (0) 1803 524238; email: k@eyeconditions.org.uk; www.eyeconditions.org.uk).

\section{The British Retinitis Pigmentosa Society}

The British Retinitis Pigmentosa Society (BRPS) was formed in 1975 to bring together people with retinitis pigmentosa and their families. The principle aims of BRPS are to raise funds to support the programme of medical research into an eventual cure for this hereditary disease, and through the BRPS welfare service, help members and their families copy with the everyday concerns caused by retinitis pigmentosa. Part of the welfare service is the telephone helpline $(+44$ (0)1280 860363 ), which is a useful resource for any queries or worries relating to the problems retinitis pigmentosa can bring. This service is especially valuable for those recently diagnosed with retinitis pigmentosa, and all calls are taken in the strictest confidence. Many people with retinitis pigmentosa have found the Society helpful, providing encouragement, and support through the Helpline, the welfare network and the BRPS branches throughout the UK. (tel: +44 (0)1280 821 334; email: lynda@brps.demon.co.uk; web site: www.brps.demon.co.uk)

\section{3rd Interdisciplinary Symposium on the Treatment of \\ Autoimmune Disorders}

The 3rd Interdisciplinary Symposium on the Treatment of Autoimmune Disorders will be held in Leipzig, Germany on the 6-8 June 2002. Topics to be covered include: basic aspects of autoimmune diseases, experimental therapeutic concepts, and clinical studies providing novel concepts or novel focus on established therapies. There will also be the presentation of the Nils-Ilja-Richter Award (application deadline is April 2002, further details on the web site). Further details: Prof. Dr. med. Michael Sticherline, Department of Dermatology, University of Leipzig (email: sticm@medizin.uni-leipzig.de; website: www.autoimmun.org); Fördergesellschaft zur Therapie von Autoimmunerkrankugen e.V. (email: autoimmun.org@gmx.de)

\section{International Society for Behçet's Disease}

The 10th International Congress on Behcet's Disease will be held in Berlin 27-29 June 2002. Further details: Professor Ch Zouboulis (email: zoubbere@zedat.fu-berlin.de).

\section{Singapore National Eye Centre 5th International Meeting}

The Singapore National Eye Centre 5th International Meeting will be held on 3-5 August
2002 in Singapore. Further details: Ms Amy Lim, Organising Secretariat, Singapore National Eye Centre, 11 Third Hospital Avenue, Singapore 16875l (tel: (65) 322 8374;fax: (65) 227 7290; email: Amy_Lim@snec.com.sg).

\section{BEAVRS Meeting}

The next BEAVRS meeting will be held in the Dalmahoy Hotel near Edinburgh on 31 October to 1 November 2002. Further details: Susan Campbell, Medical Secretary, Gartnavel General Hospital (email: susan.j.campbell.wg@ northglasgow.scot.nhs.uk).

\section{CORRECTIONS}

We regret that an error occurred in a paper that appeared in the May 2001 issue of the $B J O$ by Lauande-Pimentel et al (Discrimination between normal and glaucomatous eyes with visual field and scanning laser polarimetry measurements. 2001;85:586-91).

After revision of the paper the authors found that the published cut-off point of the structural linear discriminant formula (LDF) was incorrect.

The original formula described by LauandePimentel et al is:

LDF $=-3.131+(0.994 \times$ ellipse modulation $)-(0.017 \times$ the number $)-(0.086 \times$ average thickness $)+(0.111 \times$ ellipse average $)$

The correct suggested cut-off point, defined as abnormal, is $<0.43$ instead of $<-0.547$. The use of such formula, with the suggested cut-off point, resulted in an enhancement in the sensitivity ( sensitivity $=90.4 \%$, specificity $=82.4 \%$ ) of the scanning laser polarimeter to detect glaucomatous changes.

An error occured in the article: Additive effect of unoprostone and latanoprost in patients with elevated intraocular pressure $\mathrm{Br} J \mathrm{Oph}$ thatmol 2002:86:75-9. The authors should have been listed as Tin Aung, Paul T K Chew, Francis T S Oen, Yiong-Huak Chan, Lennard H Thean, Leonard Yip, Boon-Ang Lim, Jade Soh, and Steve K L Seah.

An error occurred in the article: Fluoxetine oral administration increases intraocular pressure. Br J Ophthalmol 1996;80:678. The authors should have been listed as C Costagliola, L Mastropasqua, L Steardo, N Testa. 